\title{
Efeitos da cinesioterapia laboral nos sintomas osteomusculares crônicos de servidores universitários de um setor da Universidade Federal do Paraná
}

\section{Effects of exercise therapy for workers with chronic osteomuscular symptoms from a sector of Federal University of Paraná}

\author{
Rogério Souza Braga1 (i) \\ Raiane da Silva Lima² (D) \\ Leticia Furman Bacil 3 (iD) \\ Ana Carolina Brandt de Macedo ${ }^{4}$ (1) \\ Arlete Ana Motter 5

\begin{abstract}
1-4Universidade Federal do Paraná (Curitiba). Paraná, Brasil. rogersbraga@hotmail.com, raiane.s.l@hotmail.com, leticiafurman17@gmail.com, acbrandtmacedo@gmail.com ${ }^{5}$ Autora para correspondência. Universidade Federal do Paraná (Curitiba). Paraná, Brasil. arlete.motter@uol.com.br
\end{abstract}

RESUMO | INTRODUÇãO: Os servidores universitários passam longos períodos sentados durante a jornada de trabalho, podendo alterar a flexibilidade e desencadear sintomas de desconforto e/ou dor osteomuscular. A cinesioterapia laboral, com exercícios físicos realizados no local de trabalho, pode ser uma estratégia importante para melhorar o conforto e prevenir os sintomas a essa população. OBJETIVO: Verificar os efeitos dos exercícios de cinesioterapia laboral na amplitude de movimento e nos sintomas osteomusculares crônicos em servidores do setor universitário. CASUÍSTICA E MÉTODOS: Trata-se de um estudo de coorte de intervenção conhecida. Realizou-se avaliação da amplitude de movimento (ADM) de ombro, quadril e tronco por meio de fleximetria e para os sintomas osteomusculares, o questionário Nórdico. A cinesioterapia laboral teve duração de 20 minutos por atendimento, duas vezes por semana, durante 7 meses. RESULTADOS: Participaram do estudo 13 servidores, de ambos os sexos, com idades entre 22 e 65 anos. Observou-se aumento significativo da amplitude de movimento de $73 \%$ nas articulações avaliadas. Após 1 mês do término da cinesioterapia laboral, realizou-se nova avaliação com a fleximetria, resultando em diminuição da amplitude em $50 \%$ das articulações. O questionário Nórdico indicou que 90\% dos participantes relataram problemas osteomusculares nos últimos 12 meses. CONCLUSÃO: A cinesioterapia laboral se mostrou eficaz nos distúrbios osteomusculares dos servidores, no aumento e manutenção da amplitude de movimento. Porém, a atividade física deve ser adotada como hábito de vida para que os resultados perpetuem.

PALAVRAS-CHAVE: Exposição ocupacional. Distúrbios osteomusculares relacionados ao trabalho. Exercícios.
ABSTRACT | INTRODUCTION: University employees spend long periods in a row during a working day, they can change flexibility and trigger symptoms of discomfort and / or musculoskeletal pain. Exercise therapy for workers, with physical exercises performed at the workplace, can be an IMPORTANT strategy to improve comfort and prevent the symptoms of this population. OBJECTIVE: To verify the effects of exercise therapy for workers on the range of motion and chronic musculoskeletal symptoms in university sector employees. CASUISTRY AND METHODS: This is a cohort study of known intervention. has been done the assessment of range of motion (ROM) of the shoulder, hip and trunk was performed using fleximetry and for musculoskeletal symptoms, or Nordic questionnaire. Labor kinesiotherapy lasted 20 minutes per visit, twice a week, for 7 months. RESULTS: 13 workers, of both sexes, aged between 22 and 65 years participated in the study. There was a significant $73 \%$ increase in ROM in the joints assessed. One month after the end of labor kinesiotherapy, a new assessment with flexometry was performed, resulting in a $50 \%$ decrease in amplitude of joints. The Nordic questionnaire indicates that $90 \%$ of the participants reported musculoskeletal problems in the last 12 months. CONCLUSION: exercise therapy for workers proved to be effective in the musculoskeletal disorders of servers, without increasing and maintaining range of motion. However, physical activity must be adopted as a life habit for the perpetuated results.

KEYWORDS: Occupational exposure. Work-related musculoskeletal disorders. Exercises. 


\section{Introdução}

A cinesioterapia laboral conhecida também como ginástica laboral, é a realização de exercícios físicos de aquecimento, fortalecimento muscular, coordenação motora, alongamento e relaxamento realizados no próprio local de trabalho, levando em conta as necessidades dos trabalhadores a quem se destina, incentivando e sensibilizando-os sobre a importância da prática de atividades físicas e de promoção em saúde, assim como a melhora no desempenho laboral'.

Com a realização da cinesioterapia laboral são esperados benefícios físicos como diminuição do sedentarismo, do estresse e melhora da qualidade de vida, mesmo que a sessões sejam consideradas "rápidas" (8 a 12 minutos) $)^{2}$. Pensando na ação em relação a prevenção de distúrbios osteomusculares, diminuição da sobrecarga emocional e física dos trabalhadores, o que se deseja alcançar com a cinesioterapia laboral é o alívio de possíveis dores corporais e consequentemente o aumento da produtividade ${ }^{2,3}$. Os fatores como o ambiente, turno, esforço nas tarefas durante o trabalho e na relação interpessoal podem afetar as condições de trabalho, assim como as cargas físicas e emocionais que são adquiridas de ambientes externos/pessoais ${ }^{4,5}$.

Quanto a importância da cinesioterapia laboral em servidores universitários, $40 \%$ dos avaliados em outra universidade pública do Paraná indicaram ter dores osteomusculares, sendo que pelo menos $5 \%$ destes consideraram a dor ou desconforto como extrema. Podemos relacionar essa dor com os movimentos repetitivos realizados durante a jornada de trabalho, ou com a postura pouco ergonômica adotada ${ }^{6}$.

Além disso, os programas de promoção à saúde são úteis para evitar problemas futuros no próprio ambiente de trabalho, pois facilita a adesão tanto da empresa quanto dos trabalhadores ${ }^{7,8}$.

Dados do Dieese ${ }^{9}$ mostram que em 2015, 44.097 pessoas que trabalhavam com administração pública em geral foram afastados do trabalho por doença profissional ou do trabalho, sendo os mais acometidos seguidos dos profissionais de tratamento e disposição de resíduos não-perigoso com 24.612 afastamentos e atividades de atendimento hospitalar, exceto pronto-socorro e unidades para atendimento a urgências com 5.345 afastamentos. Tendo em vista estas informações o objetivo deste estudo foi verificar os efeitos dos exercícios de cinesioterapia laboral na amplitude de movimento e nos sintomas osteomusculares crônicos em servidores do setor universitário.

\section{Casuística e Métodos}

Trata-se de um estudo de coorte de intervenção conhecida, transversal com delineamento descritivo, do tipo quantitativo analítico, aprovado pelo Comitê de Ética e Pesquisa da própria instituição (Parecer $n^{\circ}$ 1343168/2015 e Número do CAAE: 48185615.7.0000.0102). De acordo com o cálculo de poder amostral ( $n=Z a / 2.6 \mathrm{E})$, sendo " $n$ " para número de indivíduos, "Za/2" para valor crítico que corresponde ao grau de confiança desejado, " $\mathbf{6}$ " para desvio padrão populacional da variável estudada e "E" para margem de erro, seriam necessários 145 participantes, para um erro amostral de $5 \%$ e confiabilidade de $95 \%$, porém a amostra foi por conveniência e contou com treze servidores, sabendo-se que no setor existem aproximadamente 100 técnicos e 250 docentes. Levando em consideração os seguintes critérios de inclusão: trabalhadores de ambos os sexos, faixa etária entre 18 a 70 anos; apresentassem ou não patologias relacionadas ao trabalho; que fossem servidores exclusivamente do Setor de Ciências Biológicas da Universidade em estudo. Como critérios de exclusão: em pós-cirúrgico sem autorização médica para realizar atividade física; presença de dor aguda em ombro, quadril e coluna lombar; gestante (sem liberação devida do médico); hipertensão não controlada (sem liberação médica).

As avaliações foram realizadas no próprio local de trabalho dos servidores, levando em média $20 \mathrm{mi}$ nutos cada participante, sendo dois aplicadores, um para avaliação da fleximetria (avaliador dependente) e outro para aplicação do questionário Nórdico.

A Figura 1 ilustra a seleção, inclusão ou exclusão dos participantes. Foram identificados 350 possíveis participantes. A princípio, compareceram dezessete participantes, porém alguns desistiram e outros não alcançaram o número de 20 atendimentos de cinesioterapia laboral, durante o período em que foi aplicado ou não compareceram para a reavaliação, portanto foram excluídos do estudo quatro participantes. 


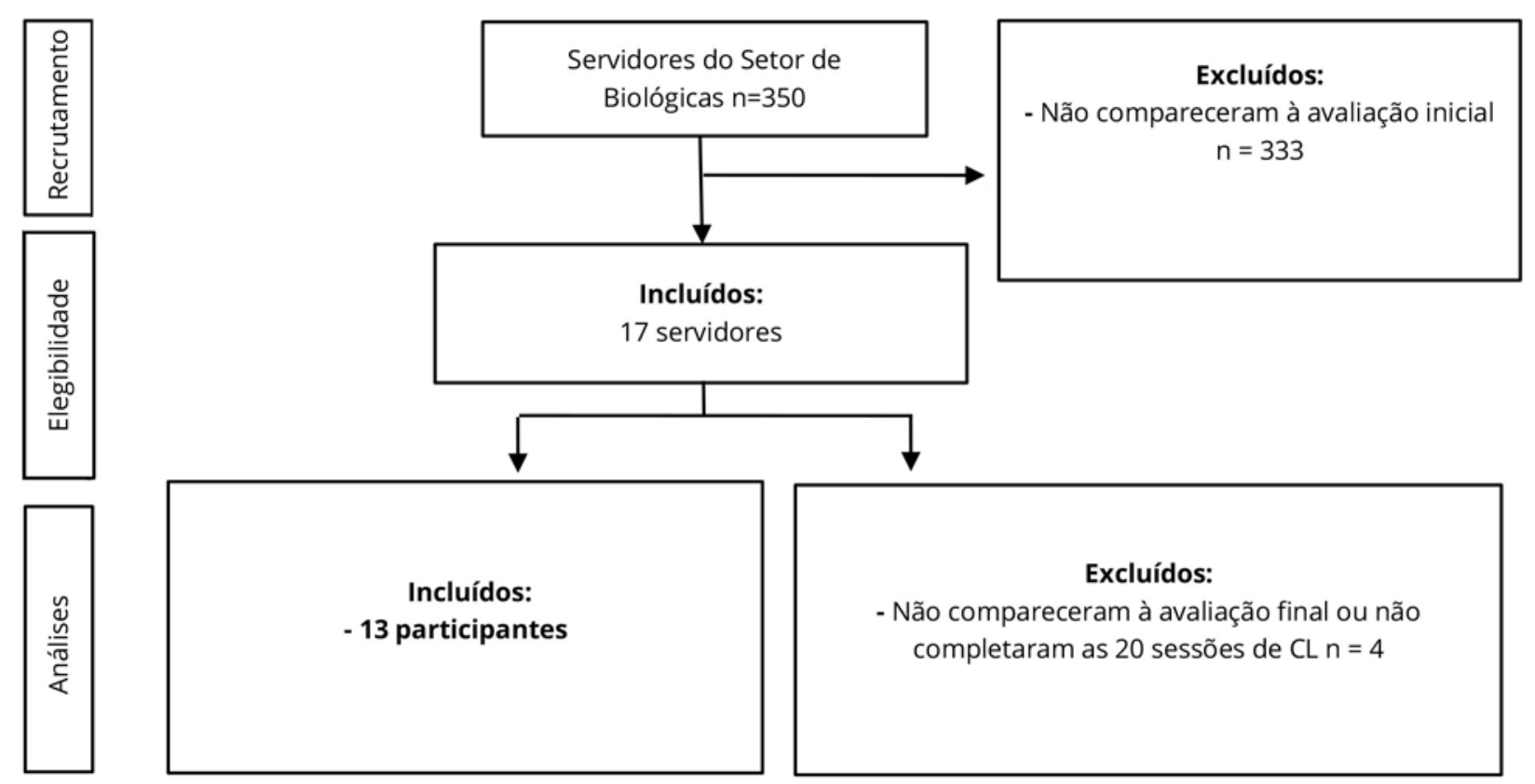

\section{Procedimentos}

As amplitudes de movimento das articulações do ombro, quadril e tronco foram avaliadas por meio de fleximetria (Sanny FL6010) ${ }^{10}$. A avaliação da ADM foi realizada por um único pesquisador previamente treinado.

Os distúrbios musculoesqueléticos foram avaliados por meio do questionário Nórdico ${ }^{11}$. O instrumento permite reconhecer o histórico de possíveis comprometimentos musculoesqueléticos, consulta com profissionais de saúde, o diagnóstico relacionado a esta consulta e possíveis limitações quanto a realização de exercícios físicos.

Os questionários foram aplicados pelo mesmo avaliador para evitar diferentes interpretações das questões e dúvidas referentes ao teor dos temas abordados.

Ao final de 20 atendimentos os instrumentos foram aplicados novamente, com a finalidade de reavaliar e mensurar os efeitos dos exercícios propostos para os participantes. Foi realizada uma nova reavaliação da fleximetria após 1 mês do término da cinesioterapia laboral, com o intuito de analisar a perduração dos efeitos após encerramento das atividades.

\section{Intervenções}

A intervenção fisioterapêutica foi realizada de abril a outubro de 2016, com duração de vinte semanas, totalizando cinco meses. Foram aplicados os exercícios cinesioterapêuticos com o grupo de participantes, duas vezes na semana, 20 minutos por atendimento, totalizando 40 sessões.

Os protocolos aplicados na cinesioterapia laboral foram elaborados pelos autores, levando em consideração as atividades laborais dos participantes e conceitos da literatura como tipo da atividade (distensionamento), tempo, local de realização e modalidade de exercícios a serem aplicados ${ }^{12}$. Os participantes realizaram as atividades propostas, na maioria das vezes, de forma individual. No entanto, em alguns momentos, os exercícios foram realizados em duplas para possibilitar interação e socialização entre os membros do grupo. Todos os atendimentos contaram com a utilização de músicas e jogo de luzes selecionados pelos pesquisadores como forma de incentivar e promover interatividade às práticas. Esses protocolos contemplaram três etapas: aquecimento, fortalecimento e relaxamento, nesta ordem, com duração aproximada de cinco minutos cada. Foram elaborados quatro protocolos, dois para as duas primeiras dez semanas e dois para as últimas dez semanas, sendo um para cada dia da semana. 
Os exercícios individuais foram compostos por aquecimento com atividades aeróbicas como danças, caminhadas, saltos, pedaladas, seguidos de fortalecimento muscular de bíceps braquial, tríceps braquial, deltoide, flexores do carpo, oblíquo externo, quadríceps, glúteos, isquiotibiais e gastrocnêmio, com três séries de 12 repetições, finalizando as atividades de alongamento com duração de 20 segundos para cada segmento e a prática de massagens, como amassamento e tapotagem, (realizadas em duplas) ${ }^{13,14,15}$.

\section{Análise estatística}

Os dados foram apresentados em média+/-desvio padrão (DP), frequencia absoluta e relativa em porcentagem. Os dados coletados foram analisados por meio de estatística descritiva através dos programas SPSS 20.0. A normalidade da amostra foi avaliada pelo teste de Shapiro-Wilk. Foi realizado o teste t de student pareado na comparação pré e pós e também após 1 mês.

\section{Resultados}

A amostra foi composta por servidores de ambos os gêneros, sendo 11 do gênero feminino e 2 do gênero masculino com idade entre 20 a 55 anos divididos em três categorias profissionais diferentes, professor (1), auxiliar administrativo (11) e técnico de laboratório (1), cada uma com suas respectivas características nas atividades de trabalho, como também horas trabalhadas na semana. Docente com 20 horas semanais, técnico de laboratório e auxiliar administrativo 40 horas semanais (Tabela 1).

Tabela 1. Tabela sociodemográfica

\begin{tabular}{|c|c|}
\hline Variáveis & Trabalhadores $(n=13)$ \\
\hline Sexo feminino & $11(84,6 \%)$ \\
\hline Sexo masculino & $2(15,4 \%)$ \\
\hline Idade (anos) & $43,30 \pm 13,97$ anos \\
\hline \multicolumn{2}{|c|}{ Dados ocupacionais } \\
\hline Horas trabalhadas/semana - docente & 20 horas \\
\hline Horas trabalhadas/semana - técnico administrativo & 40 horas \\
\hline Horas trabalhadas/semana técnico de laboratório & 40 horas \\
\hline \multicolumn{2}{|c|}{ Tipo de atividade } \\
\hline Professor & $1(7,7 \%)$ \\
\hline Auxiliar Administrativo & $11(84,6 \%)$ \\
\hline Técnico de Laboratório & $1(7,7 \%)$ \\
\hline
\end{tabular}




\section{Fleximetria}

Com a fleximetria foi possível avaliar as principais articulações: ombro, quadril e tronco.

A Tabela 2 apresenta os valores obtidos na fleximetria de ombro (pré e pós-CL). Após 1 mês sem a CL, observa-se uma diferença significativa na flexão de ombro direito e esquerdo, extensão de ombro esquerdo, adução de ombro direito e esquerdo, abdução de ombro direito e esquerdo, comparando com a pós-CL.

Tabela 2. Fleximetria do ombro dos participantes, 2016

\begin{tabular}{|c|c|c|c|c|c|}
\hline Movimento & $\begin{array}{l}\text { Pré } \\
\text { CL(média/DP) }\end{array}$ & $\begin{array}{l}\text { Pós } \\
\text { CL(média/DP) }\end{array}$ & $\mathbf{P}$ & $\begin{array}{l}\text { Após } 1 \text { mês sem } \\
\text { CL }\end{array}$ & $\mathbf{P}$ \\
\hline Flexão do ombro direito & $146,15 \pm 7,68$ & $162,31 \pm 10,33$ & $0,00^{*}$ & $157,69 \pm 8,80$ & $0,00^{*}$ \\
\hline Flexão do ombro esquerdo & $147,15 \pm 8,06$ & $161,92 \pm 9,03$ & $0,00 *$ & $157,30 \pm 7,25$ & $0,032 *$ \\
\hline Extensão do ombro direito & $32,54 \pm 10,58$ & $39,62 \pm 6,60$ & $0,001 *$ & $35,38 \pm 6,91$ & $0,003^{*}$ \\
\hline Extensão do ombro esquerdo & $34,77 \pm 11,06$ & $42,69 \pm 4,8$ & $0,017^{*}$ & $40,38 \pm 4,77$ & $0,002 *$ \\
\hline Adução do ombro direito & $27,62 \pm 12,15$ & $37,31 \pm 4,83$ & $0,002^{*}$ & $36,53 \pm 5$ & 0,001 * \\
\hline Adução do ombro esquerdo & $30 \pm 8,89$ & $37,69 \pm 5,25$ & $0,004^{*}$ & $34,23 \pm 4,49$ & $0,028^{*}$ \\
\hline Abdução do ombro direito & $122,62 \pm 23,21$ & $159,23 \pm 13,97$ & $0,00 *$ & $155,38 \pm 12,82$ & $0,00^{\star}$ \\
\hline Abdução do ombro esquerdo & $123,38 \pm 21,98$ & $158,47 \pm 18,86$ & 0,00 * & $151,53 \pm 17,48$ & $0,00 *$ \\
\hline Rotação externa do ombro direito & $79,08 \pm 10,57$ & $88,85 \pm 2,96$ & 0,001 * & $75,76 \pm 4,93$ & 0,807 \\
\hline $\begin{array}{l}\text { Rotação externa do ombro } \\
\text { esquerdo }\end{array}$ & $78,77 \pm 13,33$ & $88,08 \pm 2,53$ & $0,030 *$ & $74,23 \pm 5,34$ & 0,141 \\
\hline Rotação interna do ombro direito & $61,31 \pm 16,79$ & $83,85 \pm 5,82$ & 0,00 * & $78,46 \pm 4,27$ & 0,637 \\
\hline $\begin{array}{l}\text { Rotação interna do ombro } \\
\text { esquerdo }\end{array}$ & $63,77 \pm 16,28$ & $82,69 \pm 5,25$ & 0,00 * & $74,38 \pm 5,62$ & 0,099 \\
\hline
\end{tabular}

* $=$ Apresentam diferenças significativas $(p<0,05)$

A Tabela 3, apresenta os resultados da fleximetria do quadril (pré-CL e pós- $\mathrm{CL}$ ), onde observa-se aumento nos movimentos de rotação interna do quadril direito 45,30\% e esquerdo 32,73\%; abdução do quadril direito 33,31\% e esquerdo 32,87\%; adução do quadril direito 0\% e esquerdo 1,49\%. Ao compararmos a pós-CL e após 1 mês sem $\mathrm{CL}$, percebemos diferenças significativas (diminuição) nos movimentos de flexão do quadril direito e esquerdo, abdução do quadril direito e esquerdo. 
Tabela 3. Fleximetria do quadril dos participantes. Curitiba/PR, 2016

\begin{tabular}{|c|c|c|c|c|c|}
\hline Movimento & $\begin{array}{l}\text { Pré } \\
\text { CL(média/DP) }\end{array}$ & $\begin{array}{l}\text { Pós } \\
\text { CL(média/DP) }\end{array}$ & $\mathbf{P}$ & $\begin{array}{l}\text { Após } 1 \text { mês sem } \\
\text { CL }\end{array}$ & $\mathbf{P}$ \\
\hline Flexão do quadril direito & $72,92 \pm 22,75$ & $113,08 \pm 16,01$ & 2,48 & $101,53 \pm 13,90$ & $0,027 *$ \\
\hline Flexão do quadril esquerdo & $72,15 \pm 18,06$ & $113,08 \pm 14,65$ & 1,47 & $102,30 \pm 12,00$ & $0,016 *$ \\
\hline Extensão do quadril direito & $9,62 \pm 3,80$ & $9,62 \pm 1,39$ & 1 & $6,15 \pm 6,15$ & 0,606 \\
\hline Extensão do quadril esquerdo & $9,46 \pm 2,50$ & $9,62 \pm 1,39$ & 0,84 & $6,53 \pm 2,40$ & 0,529 \\
\hline Adução do quadril direito & $10,38 \pm 2,47$ & $14,23 \pm 2,77$ & $0,001 *$ & $8,46 \pm 2,40$ & 0,139 \\
\hline Adução do quadril esquerdo & $10,77 \pm 4,00$ & $14,23 \pm 1,88$ & $0,009 *$ & $7,69 \pm 3,30$ & 0,195 \\
\hline Abdução do quadril direito & $28,85 \pm 6,50$ & $38,46 \pm 8,99$ & $0,004 *$ & $34,61 \pm 7,20$ & $0,005 *$ \\
\hline Abdução do quadril esquerdo & $28,54 \pm 8,45$ & $37,92 \pm 10,18$ & $0,017 *$ & $36,15 \pm 3,62$ & $0,00 *$ \\
\hline Rotação externa do quadril direito & $27,23 \pm 6,46$ & $40,00 \pm 5,40$ & 1,27 & $33,84 \pm 5,82$ & 0,717 \\
\hline $\begin{array}{l}\text { Rotação externa do quadril } \\
\text { esquerdo }\end{array}$ & $25,92 \pm 5,31$ & $39,00 \pm 7,28$ & 2,31 & $35,38 \pm 5,93$ & 0,251 \\
\hline Rotação interna do quadril direito & $27,00 \pm 7,58$ & $39,23 \pm 7,87$ & $0,00 *$ & $35,38 \pm 3,79$ & 0,955 \\
\hline $\begin{array}{l}\text { Rotação interna do quadril } \\
\text { esquerdo }\end{array}$ & $29,85 \pm 4,56$ & $39,62 \pm 7,49$ & $0,00 *$ & $35,38 \pm 5,57$ & 0,092 \\
\hline
\end{tabular}

A tabela 4 mostra a média da flexão de tronco pré $\mathrm{CL}(75,5)$, pós $\mathrm{CL}(90,0)$, o que corresponde a 19,20\%; após 1 mês sem $\mathrm{CL}$, este número reduziu para 80,76.

Tabela 4. Fleximetria do tronco dos participantes

\begin{tabular}{llllc}
\hline Movimento & Pré & Pós & P & \multicolumn{1}{c}{$\begin{array}{l}\text { Após 1 mês sem } \\
\text { CL }\end{array}$} \\
\hline Flexão de tronco & $75,5 \pm 12,3$ & $90,0 \pm 5,0$ & $0,00 *$ & $80,76 \pm 5,34$ \\
Extensão do tronco & $25,8 \pm 8,4$ & $40,4 \pm 4,8$ & 1,47 & $38,07 \pm 4,80$ \\
\hline
\end{tabular}




\section{Questionário Nórdico}

Os resultados alcançados com o questionário Nórdico encontram-se na Figura 2.

Figura 2. Resultados do questionário nórdico dos participantes, 2016

\begin{tabular}{|c|c|c|c|c|c|c|c|c|}
\hline \multirow[t]{3}{*}{ Estruturas } & \multicolumn{2}{|c|}{$\begin{array}{l}\text { Nos últimos } 12 \text { meses } \\
\text { teve problemas em: }\end{array}$} & \multicolumn{2}{|c|}{$\begin{array}{l}\text { Foi impedido de } \\
\text { realizar atividades } \\
\text { normais }\end{array}$} & \multicolumn{2}{|c|}{$\begin{array}{l}\text { Consultou profissional } \\
\text { da saúde }\end{array}$} & \multicolumn{2}{|c|}{$\begin{array}{l}\text { Nos últimos } 7 \text { dias } \\
\text { teve problema em: }\end{array}$} \\
\hline & Antes & Depois & Antes & Depois & Antes & Depois & Antes & Depois \\
\hline & $n(\%)$ & $n(\%)$ & $n(\%)$ & $n(\%)$ & $n(\%)$ & $n(\%)$ & $n(\%)$ & \\
\hline oço & $7(53,8)$ & $5(38,4)$ & $1(7,7)$ & 0 & $2(15,4)$ & $2(15,4)$ & $4(30,7)$ & $1(7,7)$ \\
\hline Ombro & $7(53,8)$ & $4(30,7)$ & $1(7,7)$ & 0 & $3(23)$ & $2(15,4)$ & $5(38,4)$ & $2(15,3)$ \\
\hline Tórax & $9(69,2)$ & $4(30,7)$ & $1(7,7)$ & 0 & $2(15,3)$ & $1(7,6)$ & $5(38,4)$ & 0 \\
\hline & $3(23)$ & $2(15,3)$ & 0 & 0 & 0 & 0 & $1(7,7)$ & 0 \\
\hline & $3(23)$ & $4(30,7)$ & 0 & 0 & $1(7,7)$ & $2(15,3)$ & $3(23)$ & $2(15,3)$ \\
\hline & $7(53,8)$ & $6(46,1)$ & $2(15,4)$ & 0 & $1(7,7)$ & $1(7,7)$ & $4(30,7)$ & $1(7,7)$ \\
\hline Quadril/coxas & $7(53,8)$ & $4(30,7)$ & $2(15,4)$ & 0 & $2(15,3)$ & $1(7,7)$ & $4(30,7)$ & $1(7,7)$ \\
\hline Joelhos & $5(38,4)$ & $4(30,7)$ & $2(15,4)$ & $1(7,6)$ & $2(15,3)$ & $2(15,3)$ & $3(23)$ & $2(15,3)$ \\
\hline Tornozelos/pés & $3(23)$ & $2(15,3)$ & 0 & 0 & $1(7,7)$ & $1(7,7)$ & $3(23)$ & $1(7,7)$ \\
\hline
\end{tabular}

$\mathrm{Na}$ avaliação pelo questionário Nórdico, foi possível observar que $90 \%$ dos participantes relataram problemas osteomusculares nos últimos 12 meses. Sendo que a maioria relatou problemas na região do pescoço, ombro, lombar, quadril/coxas e principalmente do tórax $(69,2 \%)$ diminuindo para 30,7\% após. As regiões menos acometidas foram pés/tornozelos, punhos/mãos e cotovelo.

\section{Discussão}

Os participantes do estudo eram relativamente jovens, principalmente mulheres, com carga horária semanal predominantemente de 40 horas, em sua maioria auxiliares administrativos.

No estudo, foi possível observar que houve melhora significativa na articulação do ombro em todos os movimentos avaliados. Após 1 mês sem a CL, observou-se uma diferença significativa na flexão de ombro direito e esquerdo, extensão de ombro esquerdo, adução de ombro direito e esquerdo, abdução de ombro direito e esquerdo, comparando com a pós CL.
Ao compararmos a pós- $\mathrm{CL}$ e após 1 mês sem $\mathrm{CL}$ na articulação do quadril, percebemos diferenças significativas (diminuição) nos movimentos de flexão do quadril direito e esquerdo, abdução do quadril direito e esquerdo, como também,em flexão de tronco.

$\mathrm{Na}$ avaliação, as articulações do ombro, quadril e tronco, grande parte dos participantes apresentaram resultados abaixo do valor de referência encontrado na literatura. Nos dados apresentados na reavaliação, é possível observar importante melhoria nas articulações citadas acima.

Um mês após o término da cinesioterapia laboral, os participantes apresentaram resultados abaixo do valor de referência (50\%), comparados com a reavaliação, no período da cinesioterapia laboral

Na maioria das empresas a pausa já existe, o que se faz necessário é a justificativa por meio de benefícios, que muitas vezes não aparecem de imediato em ganhos econômicos ou aumento da produtividade, mas com aumento da disposição para a atividade laboral, da satisfação, do clima organizacional, do relacionamento social e do estímulo a bons hábitos para cultivar a qualidade de vida destas pessoas ${ }^{14}$. 
O principal fator positivo alcançado pelas intervenções com a cinesioterapia laboral, neste estudo, foi o aumento da amplitude de movimento. Esses resultados são de extrema importância, tendo em vista que as amplitudes de movimento das articulações do corpo em medidas funcionais são necessárias na execução de atividades de vida diária, na prática de exercícios, e sobretudo na prevenção de lesões ${ }^{15}$.

Um fator negativo foi que, após um mês do término da cinesioterapia laboral, os participantes apresentaram diminuição da amplitude de movimento. Quando diminuída, pode levar a limitações no movimento provocando dores e gerando demais sintomas osteomusculares ${ }^{16,17}$.

A CL quando aplicada em professores em sessões de 20 minutos, dividindo o tempo em preparatória compensatória e relaxamento, trouxe melhora do rendimento e bem-estar, assim como a melhor integração entre colegas de trabalho ${ }^{18}$.

A mesma quando realizada em enfermeiros e auxiliares de enfermagem de dois hospitais de Barcelona, que a ergonomia, a promoção de um estilo de vida saudável e um programa de prevenção específico de lesões foi eficaz para diminuir as dores nas regiões de pescoço, ombro, cervical e lombar, semelhante aos resultados obtidos no nosso estudo ${ }^{12}$.

Os estudos mostraram variáveis complexas, como quadro álgico, estilo e qualidade de vida, componentes relacionados à saúde mental, entre tantos outros, geralmente associados aos resultados das intervenções da cinesioterapia laboral. Os desenhos quantitativos são capazes de fornecer dados para a análise da eficácia e eficiência da terapia utilizada. Porém, para temas dessa modalidade, a literatura sugere análises qualitativas e de corte participativo, nas quais a opinião dos trabalhadores pode ser ouvida de maneira abrangente e inclusiva ${ }^{19}$.

A pesquisa citada utilizou uma técnica diferente da deste estudo, aplicando os exercícios que compõem a cinesioterapia separadamente durante o período de realização. Os atendimentos tiveram o objetivo de prevenir dores crônicas, com três meses de duração, cinco intervenções semanais de ginástica de aquecimento (10-15min diários), cinco minutos de ginástica de pausa (10-15min diários), ambas no ambiente de trabalho, bem como três atendimentos semanais de ginástica compensatória (30-60min por sessão). Após concluímos que o número de pontos dolorosos no público estudado havia diminuído, permanecendo apenas dores nos carpos. Mesmo assim, a atividade física dentro da empresa mostrou-se um forte auxiliar na manutenção da qualidade do trabalho ${ }^{20}$.

O estudo citado correlacionou a incidência de cansaço mental e o número de queixas osteomusculares, expondo que trabalhadores que relataram cansaço mental apresentaram maior número de queixas osteomusculares, concluindo assim que os dois aspectos muitas vezes estão interligados, mostrando a importância de intervenções que venham proporcionar ao trabalhador melhor qualidade na atividade laboral e também em aspectos psico-cognitivos, os quais vão refletir tanto na produtividade quanto na qualidade de vida desses indivíduos ${ }^{21}$.

Uma das enfermidades mais acometidas por trabaIhadores que permanecem um período longo sentado, como é o caso da população estudada, é a lombalgia. Em estudo, aplicou-se um programa de dez atendimentos de cinesioterapia laboral, de dez minutos, duas vezes por semana, durante cinco semanas. $O$ resultado da pesquisa mostrou que a cinesioterapia laboral melhorou a dor lombar, diminuindo a sua intensidade, melhorando a capacidade funcional dos músculos estabilizadores do tronco e a amplitude de movimento articular ${ }^{22}$.

A literatura citada corrobora este estudo em relação a dificuldade na adesão de participantes, indicando maior número de intervenções e assim abrangendo mais locais de trabalho e trabalhadores ${ }^{14}$.

Além disto, a realização de atividade física produz efeitos neuroprotetores por induzirem maior secreção de fatores neurotróficos ligados à glia, agindo positivamente na neuroproteção e na neuroplasticidade de neurônios dopaminérgicos ${ }^{23}$. Os autores da pesquisa citada, relatam os efeitos da atividade física na promoção e integração de vários impulsos neuronais cerebrais, permitindo mudanças plásticas que beneficiam na prevenção de lesões e ação positiva em estruturas já lesionadas ${ }^{24}$. 
A amostra de conveniência não chegou a 10\% da população do setor universitário estudado, constituindo-se numa limitação da pesquisa, portanto, novas abordagens precisariam ser realizadas com uma amostra maior. Nesse estudo não foi realizada análise descritiva dividindo os participantes que fizeram ou não a interação e seus diferentes desfechos. Assim, sugere-se que para futuros estudos $O$ protocolo de pesquisa seja único para evitar variações entre os indivíduos.

\section{Conclusão}

A partir dos resultados alcançados pelo estudo, foi possível observar que a cinesioterapia laboral se mostrou um método eficaz de intervenção terapêutica na promoção de enfermidades ocasionadas por má postura, imobilização, gestos repetitivos e manutenção da amplitude de movimento.

Como grande parte dos participantes trabalha em atividades de escritório ou em laboratórios, ambientes propícios para movimentos repetitivos, minuciosos, compensações posturais, que podem acarretar distúrbios musculoesqueléticos e psicossociais é importante uma abordagem profissional que vise prevenir ou tratar esses sintomas.

Desta forma, foi possível constatar aumento na flexibilidade dos participantes com a realização da cinesioterapia laboral duas vezes na semana com duração de 20 minutos. Para obter melhores resultados, sugere-se um número maior de participantes e maior número de atendimentos, além de investigações que avaliem a qualidade de vida desses trabalhadores, maior quantidade de intervenções semanais, maior número de atendimentos, além de incentivos acerca da realização da ginastica laboral, expondo seus benefícios para os trabalhadores e para a produtividade da empresa em si.

\section{Contribuições dos autores}

Braga RS, Lima RS participaram da concepção, delineamento, aquisição de TCLE, coleta e análise de dados da pesquisa, interpretação de resultados e redação do artigo científico. Bacil LF participou da revisão do artigo científico, atualização da literatura, correções e tradução. De Macedo ACB, Motter AA participaram da concepção, delineamento, análise e interpretação de resultados, revisão do artigo científico e encaminhamento.

\section{Conflitos de interesses}

Nenhum conflito financeiro, legal ou político envolvendo terceiros (governo, empresas e fundações privadas, etc.) foi declarado para nenhum aspecto do trabalho submetido (incluindo, mas não se limitando a subvenções e financiamentos, participação em conselho consultivo, desenho de estudo, preparação de manuscrito, análise estatística, etc.).

\section{Referências}

1. Fernandes CHA, Santos PVS. Ergonomia: Uma revisão da literatura acerca da ginástica laboral. Nucleus. 2019;16(2):211-220. doi: $\underline{10.3738 / 1982.2278 .3598}$

2. lackstet L, Gonçalves ACBF, Soares SFC. Análise dos benefícios da cinesioterapia laboral a curto, médio e longo prazo: uma revisão de literatura. Arch Health Invest. 2018;7(5):168-173. doi: 10.21270/archi.v7i5.2990

3. Serra MVGB, Camargo PR, Zaia JE, Tonello MGM, Quemelo PRV. Effects of physical exercise on musculoskeletal disorders, stress and quality of life in workers. Int J Occup Saf Ergon. 2016;24(1):1-6. doi: $10.1080 / 10803548.2016 .1234132$

4. Cullen KL, Irvin E, Collie A, Clay F, Gensby U, Jennings PA et al. Effectiveness of workplace interventions in return-to-work for musculoskeletal, pain-related and mental health conditions: an update of the evidence and messages for practitioners. J Occup Rehabil. 2018;28(1):1-15. doi: 10.1007/s10926-016-9690-x

5. Cardoso VF, Pizzol RJ, Takamoto PM, Gobbo LA, Almeida ALJ. Asociación del diagnóstico clínico con situación laboral de usuarios de servicio de fisioterapia. Rev Fisioter Pesqui. 2017;24(2):169-175. doi: 10.1590/1809-2950/16846124022017

6. Ramos, LS, Weiber EA, Santos CB, Oliveira HFDR. Análise da incidência de LER/DORT em colaboradores que participam da ginástica laboral. Anais do $17^{\circ}$ CONEX - Encontro Conversando sobre Extensão na UEPG e do $2^{\circ}$ EAEX - Encontro Anual de Extensão Universitária, 2019; Ponta Grossa - PR. 
7. Manosso M, Lanferdini FJ, Dal'Agnol MJ, Roncada C, Dias CP. Comparação dos níveis de estresse e estilo de vida entre praticantes e não praticantes de ginástica laboral. Revisa Brasileira de Ciência e Movimento. 2014;22(2):65-71. doi: 10.18511/rbcm.v22i2.3961

8. Moreto AF, Chesani FH, Grillo LP. Sintomas osteomusculares e qualidade de vida em costureiras do município de Indaial, Santa Catarina. Fisioter Pesqui. 2017;25(2):163-168. doi: 10.1590/18092950/16833624022017

9. Departamento Intersindical de Estatística e Estudos Socioeconômicos. Anuário da saúde do trabalhador. São Paulo: DIEESE; 2016.

10. Renner JS. Prevenção de distúrbios osteomusculares relacionados ao trabalho. Boletim da saúde. 2005;19(1):73-80.

11. Pinheiro FA, Troccoli BT, Carvalho CV. Validação do Questionário Nórdico de Sintomas Osteomusculares como medida de morbidade. Rev Saúde Pública. 2002;36(3):307-12. doi: $\underline{10.1590 / 50034-89102002000300008}$

12. Soler-Font M, Ramada JM, van Zon SK, Almansa J, Bültmann $\mathrm{U}$, Serra $\mathrm{C}$ et al. Multifaceted intervention for the prevention and management of musculoskeletal pain in nursing staff: Results of a cluster randomized controlled trial. PloS One, 2019;14(11):e0225198. doi: 10.1371/journal.pone.0225198

13. Correia OD, Oliveira ESW, Teixeira KVS, Carvalho MF, Vieira RBR, Moreira RGS. Exercícios terapêuticos na dor crônica. Cadernos de educação, saúde e fisioterapia. 2018;5(10).

14. Teixeira GM, Costa MAM, Melo DCS, Florentino MP, Albuquerque KCTM, Fernandes MG et al. Ginástica laboral: compreendendo a baixa adesão pela óptica do funcionário. Fisioterapia Brasil. 2016;17(1):37-40.

15. Kisner C, Colby LA. Exercícios Terapeuticos- Fundamentos e técnicas. São Paulo: Manole; 2016.

16.So BCL, Szeto GPY, Lau RWL, Dai J, Tsang SMH. Effects of Ergomotor Intervention on Improving Occupational Health in Workers with Work-Related Neck-Shoulder Pain. International Journal of Environmental Research and Public Health.

2019;16(24):E5005. doi: 10.3390/ijerph16245005

17. Pescatello LS, American College of Sports Medicine. ACSM's guidelines for exercise testing and prescription. 9.ed. Philadelphia: Wolters Kluwer/Lippincott Williams \& Wilkins Health; 2014.

18. Bezerra GMR, Félix KD. Os benefícios da ginástica laboral para prevenção de dores osteomusculares em professores da escola Gesner Teixeira/Gama-DF. Revista de Saúde da Faciplac. 2015;2(1):40-50.
19. Neves RF, Araujo SPA, Magalhães LV, Lima MAG. A ginástica laboral no Brasil entre os anos de 2006 e 2016. Rev Bras Med Trab. 2018;16(1):82-96. doi: 10.5327/Z1679443520180078

20. Silvera JWP, Laat EF, Pilatti LA, Silva F, Machowski CM, Samila, G. Ginástica laboral e prevenção de dores crônicas. Cinergis, 2013;14(2):115-119. doi: 10.17058/cinergis.v14i2.3099

21. Motter AA, Santos M, Guimarães ATB. O que está a sombra na carga de trabalho de estivadores? Rev. Produção online. 2015;15(1):321-344. doi: 10.14488/1676-1901.v15i1.1845

22. Laux RC, Pagliari P, Effting Junior JV, Corazza ST. Programa de ginástica laboral e a redução de atestados médicos. Rev Cienc Trab. 2016;18(56):130-133. doi: 10.4067/S0718$\underline{24492016000200009}$

23. Cruz JM. Benefícios da caminhada na água na qualidade de vida de pacientes com doença de Parkinson. [trabalho de conclusão de curso]. Rio Grande do Norte: Universidade Federal do Rio Grande do Norte; 2016.

24. Damazio LCM, Melo RTR, Lima MC, Pereira VG, Ribeiro RIMA, Alves NR et al. Exercício físico promove neuroproteção estrutural e funcional em ratos com isquemia cerebral. Rev Neurociec. 2015;23(4):581-588. doi: 10.34024/rnc.2015.v23.7978 